\title{
Theta- and alpha-band EEG activity in response to eye gaze cues in early infancy
}

\author{
Christine Michel ${ }^{\mathrm{a}, *}$, Manuela Stets ${ }^{\mathrm{b}}$, Eugenio Parise ${ }^{\mathrm{c}}$, Vincent M. Reid ${ }^{\mathrm{c}}$, Tricia Striano ${ }^{\mathrm{d}, \mathrm{e}}$, Stefanie Hoehl ${ }^{\mathrm{a}}$ \\ a Institute of Psychology, Heidelberg University, Hauptstrasse 47-51, 69117 Heidelberg, Germany \\ b Department of Psychological and Brain Sciences, Indiana University, 1101 E. 10th St., Bloomington, IN 47405, USA \\ c Department of Psychology, Lancaster University, Bailrigg, Lancaster, United Kingdom \\ d Hunter College, 695 Park Avenue, New York, NY 10065, USA \\ e The Institute for Education on Health and Research, Milton, MA, USA
}

Keywords:

Infancy

Eye gaze cues

Theta synchronization

Alpha desynchronization

\begin{abstract}
A B S T R A C T
In order to elucidate the development of how infants use eye gaze as a referential cue, we investigated theta and alpha oscillations in response to object-directed and object-averted eye gaze in infants aged 2, 4, 5, and 9 months. At 2 months of age, no difference between conditions was found. In 4- and 9-month-olds, alpha-band activity desynchronized more in response to faces looking at objects compared to faces looking away from objects. Theta activity in 5-month-old infants differed between conditions with more theta synchronization for objectaverted eye gaze. Whereas alpha desynchronization might reflect mechanisms of early social object learning, theta is proposed to imply activity in the executive attention network. The interplay between alpha and theta activity represents developmental changes in both kinds of processes during early infancy.
\end{abstract}

\section{Introduction}

From very early on in life, eye gaze is an important cue influencing infants' perception and attention. As it helps infants to direct their attention to relevant information in the environment, eye gaze direction, among other social cues (Bertenthal et al., 2014), affects information processing and facilitates social learning (Csibra and Gergely, 2006; Hoehl et al., 2009; Reid and Striano, 2007). Here, we measure oscillatory brain activity in response to eye gaze as a referential cue in early infancy.

Infants show an early sensitivity to eye gaze direction in relation to the location of objects. Nine-month-old infants look longer to objectdirected gaze shifts than to non-object-directed gaze shifts (Senju et al., 2008). Even younger infants differentiate between objectdirected and object-averted eye gaze: event-related potentials (ERPs) in response to faces looking toward objects were compared to those for faces looking away from objects in 2-, 4- and 5-month-olds (Hoehl et al., 2008; Hoehl et al., 2009). Whereas no effects on the negative central $(\mathrm{Nc})$ component were found in the youngest age group, infants at 4 and 5 months showed a larger amplitude for this component for objectaverted gaze. As the Nc component is related to attention (Reynolds and Richards, 2005), it was concluded that infants allocated more attention

\footnotetext{
* Corresponding author.

E-mail addresses: christine.michel@psychologie.uni-heidelberg.de (C. Michel), mstets@indiana.edu (M. Stets), eugenioparise@tiscali.it (E. Parise),v.reid@lancaster.ac.uk (V.M. Reid), tstriano@hunter.cuny.edu (T. Striano), stefanie.hoehl@psychologie.uni-heidelberg.de (S. Hoehl).
}

to faces that looked away from objects because this situation was less expected and more ambiguous to them. Moreover, it was only in the 4- and not in the 5-month-olds that a larger positive slow wave (PSW) was found for object-directed looks. The PSW is related to memory updating processes (Nelson, 1997; Webb et al., 2005). Thus, eye gaze may have facilitated building memory representations for cued objects. In the aforementioned cross-sectional approach, the studies by Hoehl et al. $(2008,2009)$ highlight developmental changes in the way infants process eye gaze and its relation to objects.

Similar developmental changes have been revealed by behavioral studies. At 3 months of age, infants are already sensitive to triadic interactions (Striano and Stahl, 2005). Their ability to follow gaze shifts of strangers increases between 4 and 6 months (Gredebäck et al., 2010). At the same time, infants' joint attention skills gradually develop (Striano and Bertin, 2005) and their ability to use social cues to encode new information advances. In a live paradigm measuring looking times, infants at 7 and 9 months but not at 4 and 5 months of age showed enhanced object processing in a joint attention situation (Cleveland et al., 2007; Cleveland and Striano, 2007). Studies that presented similar stimuli on a screen found that infants were already able to use social cues for object learning at 4 months (Hoehl et al., 2014b; Reid and Striano, 2005; Reid et al., 2004; Wahl et al., 2013). These studies compared ERPs and looking times in response to objects that were previously cued by another person's eye gaze and/or head turn with objects that were not cued. Cued objects were processed more efficiently whereas uncued objects were more novel to infants when they were presented to the infant a second time. This was reflected in enhanced amplitudes of either the 
PSW or the Nc as well as in longer looking times to previously uncued objects. Eye gaze cues guided infant attention and thereby facilitated object learning. The age discrepancy between live and video-based studies may be due to the different types of paradigms and dependent variables. A video-based presentation condenses information on a small screen and this may help infants to focus on the stimuli. The setting in a live paradigm is more complex as infants are interacting with a real person who, inevitably, covers more space. Furthermore, the dependent variable in the live studies was the overt behavior of the infant, whereas video-based studies mostly applied ERPs and/or eye tracking.

The aforementioned studies show developmental changes in the way infants make use of social cues. One possible mechanism behind these changes is how infants are able to control their attention. At 4 months of age, infants supposedly react to eye gaze cues due to an automatic shift of attention (Hoehl et al., 2014b; Moore and Corkum, 1998). During the following two months, it has been proposed that an attention network starts to monitor and integrate infants' own and others' gaze direction and behavior. Between 7 and 9 months, infants are able to internally control their shifts of attention (Mundy and Newell, 2007; Petersen and Posner, 2012).

As the results of studies investigating the use of social cues differ depending on the paradigm used, the current study makes use of the same paradigm for all age groups in a cross-sectional design with infants aged 2, 4, 5, and 9 months. As in the study by Hoehl et al. (2008), infants saw static images of faces either looking toward or away from an object while their EEG was measured. So far, the neural processing of eye gaze-object relations in infancy has only been investigated using ERPs. In the current study, we analyze oscillatory changes to further clarify underlying neural mechanisms of how social information is processed.

Based on the literature, the alpha- and the theta-band are likely to be sensitive to eye gaze-object relations: theta-band activity in adults lies between 4 and about $7 \mathrm{~Hz}$ (Klimesch, 1999; Saby and Marshall, 2012). Theta in infants, that we refer to in the current study, is primarily defined between 3 and $6 \mathrm{~Hz}$ and the frequency range does not seem to change between 4 and 12 months (Saby and Marshall, 2012; Stroganova and Orekhova, 2007). Theta synchronization may imply activity of the frontal cortex including an attention network involved in executive and voluntary control of attention as it has been proposed by Posner and Petersen (Bazhenova et al., 2007; Orekhova et al., 1999; Petersen and Posner, 2012; Posner and Petersen, 1990). It has been suggested that this attention system emerges at around 4-6 months and allows infants to monitor the relation between their own and others' gaze direction and goal-directed behavior (Mundy and Newell, 2007). Frontal theta activity decreases with age. This decrease is proposed to reflect maturation processes in the attention system as the system gets increasingly effective (Orekhova et al., 1999). If theta activity implies executive control of attention, it would be expected to vary with developmental changes in response to social cues. Therefore, we expect to find no differences between conditions in theta synchronization in the 2- and 4-month-olds as the executive attention network should not be developed yet. In 9-month-olds, the network should have matured and be more efficiently functioning (Orekhova et al., 1999). As theta decreases in older infants, we expect little or no difference in theta synchronization between conditions. Changes in theta activity may reflect the development of this system which occurs at around 5-6 months of age and we therefore expect theta effects specifically in this age group.

Alpha desynchronization in adults has been related to attentional mechanisms that actively suppress distracting information to focus on relevant input (Ward, 2003). In a live triadic joint attention interaction, Lachat et al. (2012) reported attenuated alpha signal power $(11-13 \mathrm{~Hz})$ in adult participants that jointly attended to the same stimulus. This result was interpreted as reflecting higher arousal induced by mutual attentiveness. Hoehl et al. (2014a) recently showed similar effects in 9-month-old infants in a live paradigm. Here, alpha $(5-7 \mathrm{~Hz})$ desynchronized in response to novel objects only when these objects were presented in a joint attention situation (Hoehl et al., 2014a), indicating that alpha-band activity varied depending on the social context in which stimuli were perceived. Alpha desynchronization was therefore suggested to relate to early social learning processes in infants (Hoehl et al., 2014a). Enhanced alpha desynchronization may indicate that attention is focused on the relevant object (here an object that is cued by eye gaze). Thereby it could enable or at least facilitate object learning in such situations. Similar processes might already take place at 4 months as infants differentiate between eye gazes toward and away from objects and build stronger memory representations for cued objects (Hoehl et al., 2008; Hoehl et al., 2014b; Reid and Striano, 2005; Reid et al., 2004; Wahl et al., 2013). In the current study, eye gaze that is directed toward an object identifies it as an object that is of high relevance for the infant. Thus, we expect desynchronization to occur in response to object-directed gaze starting at 4 months of age in the alpha-band frequency range $4-10 \mathrm{~Hz}$, which is the typical range for alpha in infants (Marshall et al., 2002; Stroganova et al., 1999).

The current study investigates oscillatory brain activity in response to object-directed and object-averted eye gaze for synchronization in the theta range and for desynchronization in the alpha range. By studying 2-, 4-, 5-, and 9- month-old infants with the same paradigm, we expect to gain insights into how the processing of social cues develops and how attentional and social information processes change in early infancy (Cleveland et al., 2007; Cleveland and Striano, 2007; Striano and Stahl, 2005).

\section{Method}

\section{Participants}

The final sample consisted of 58 (32 female) 2-, 4-, 5-, and 9-monthold infants born full term (37-41 weeks) and within the normal range for birth weight (see Table 1 for detailed information about age, sex, and the number of trials included in the final analyses separately for each age group).

Another 79 infants were tested but excluded from the final sample due to fussiness (17) or failure to reach the minimum criterion of 10 artifact-free trials per condition (62). This inclusion criterion and the attrition rate of $58 \%$ are similar to other infant EEG studies (e.g. Elsabbagh et al., 2009; Southgate et al., 2008). Data from 14 additional infants were distorted due to technical problems and, therefore, not analyzed. The group of 4-month-old infants consists of the sample reported in Hoehl et al. (2008) and the group of 2- and 5-month-olds of the sample reported in Hoehl et al. (2009). Both of these studies investigated ERP effects. On average, infants contributed 20 artifact-free trials to the grand average per condition.

\section{Stimuli}

Static portrait photographs of two female actors served as stimuli. Their eye gaze was shifted either to the left or to the right and a colorful object was presented next to the face on one side at the height of the pupils approximately $2 \mathrm{~cm}$ away from the eyes. Consequently, two different conditions were created: in the object-directed condition, the actor looked at the object and in the object-averted condition, the actor looked away from the object (see Fig. 1). Stimuli were $19.5 \mathrm{~cm}$ (12.4 ${ }^{\circ}$ visual angle) high and $25 \mathrm{~cm}$ (15.8 visual angle) wide measured from the ear of the actor to the end of the object on the opposite side.

\section{Procedure}

During testing, infants sat on their mother's lap while their EEG was recorded continuously and their behavior was filmed for offline coding. Stimuli were presented on a $70 \mathrm{~Hz} 17$ " screen at $90 \mathrm{~cm}$ viewing distance in a dimly lit, sound-attenuated, and electrically shielded cabin. 
Table 1

Sample information and overview of included trials per condition.

\begin{tabular}{|c|c|c|c|c|}
\hline & 2-month-olds & 4-month-olds & 5-month-olds & 9-month-olds \\
\hline$N$ & 14 & 16 & 16 & 12 \\
\hline Sex & 10 female & 8 female & 7 female & 7 female \\
\hline Mean age (mm.dd) & 02.23 & 04.02 & 05.19 & 08.28 \\
\hline Age range (mm.dd-mm.dd) & $02.07-03.00$ & 03.21-04.09 & $05.02-05.29$ & 08.21-09.09 \\
\hline Mean number; standard deviation (range) of included trials: object-directed condition & $27 ; 16(10-63)$ & $19 ; 7(10-37)$ & $19 ; 10(10-41)$ & $14 ; 3(10-20)$ \\
\hline Mean number; standard deviation (range) of included trials: object-averted condition & $27 ; 16(10-62)$ & $19 ; 8(10-37)$ & $20 ; 10(10-41)$ & $14 ; 3(10-20)$ \\
\hline
\end{tabular}

A trial consisted of a central attractor (a small triangular object) presented at the center of the screen for $500 \mathrm{~ms}$ followed by a stimulus image presented for $1000 \mathrm{~ms}$. Before the next trial started, a white screen was presented with a random interval of $800-1000 \mathrm{~ms}$ (see Fig. 1). Conditions were presented in a randomized order with the constraint that each condition was not presented more than twice in a row and the number of object-directed and object-averted pictures was balanced every 20 trials. A maximum number of 200 trials (100 per condition) was presented as long as the infant looked attentively to the screen. Testing was paused or stopped if the infant became fussy or inattentive to the screen.

\section{EEG recording and analyses}

EEG was recorded continuously during testing with $19 \mathrm{Ag}-\mathrm{AgCl}$ electrodes arranged according to the 10-20 system. Data were amplified via a Twente Medical System 32-channel REFA amplifier and sampling rate was set at $250 \mathrm{~Hz}$. Data were analyzed using the custom-made scripts collection "WTools" (available on request) and EEGLab (v. 10.2.5.5a). EEG was referenced to the vertex $(\mathrm{Cz})$. Horizontal and vertical electrooculograms (EOG) were recorded bipolarly. Data were re-referenced offline to the averaged mastoids and were bandpass filtered from 2 to $65 \mathrm{~Hz}$. The EEG signal was segmented into epochs of $-1200 \mathrm{~ms}$ to $2000 \mathrm{~ms}$ around the onset of the stimulus. EEG data were rejected offline whenever the standard deviation within a $200 \mathrm{~ms}$ gliding window exceeded $80 \mu \mathrm{V}$ at any electrode (Hoehl et al., 2008). Artifacts caused by eye movement were rejected based on EOG measures. Infants' looking behavior was coded offline based on the recorded videos. Trials in which the infant did not attend to the screen were removed manually. Given that infants overtly shifted their eye gaze during the presentation of the stimulus image in only $7.97 \%$ of all presented trials, we did not analyze this behavior further.

Time-frequency analyses were conducted performing a continuous wavelet transformation. Complex Morlet wavelets were computed at $1 \mathrm{~Hz}$ frequency intervals for the frequency range 2-60 Hz. Total spectral activity was calculated performing convolutions with the wavelets on all channels. The absolute value of the result was computed and served as the dependent variable. The transformed epochs were averaged for each condition (see Csibra et al., 2000; Hoehl et al., 2014a; Parise and Csibra, 2013). Furthermore, $1000 \mathrm{~ms}$ at the beginning and at the end of each segment were removed to avoid distortions due to the transformation. Baseline correction was performed at each frequency by subtracting the mean activity of 200 ms before stimulus onset from the signal.

The grand average was calculated for both conditions for each age group separately. The time-frequency range for statistical analyses for the theta and the alpha frequency range was based on visual inspection of the data and existing literature.

\section{Theta activity}

Visual inspection of the data revealed differences between conditions mainly in the lower frequency range. The theta 1 sub-band was defined as ranging between 3.6 and $4.8 \mathrm{~Hz}$ with a peak at $4.4 \mathrm{~Hz}$ (Orekhova et al., 2006). Theta activity in this frequency range is more pronounced on frontal channels (Orekhova et al., 1999, 2006; Stroganova et al., 1998). Compared to alpha activity, the theta frequency range does not seem to shift with age (Saby and Marshall, 2012; Stroganova and Orekhova, 2007). Thus, the mean amplitude at $4 \mathrm{~Hz}$ at 400-800 ms after stimulus onset on fronto-central electrodes (F3, Fz, F4, FC3, and FC4) served as the dependent variable for all age groups (Orekhova et al., 2006).

\section{Alpha activity}

In infancy, alpha occurs on posterior-occipital channels in the frequency range 4-10 $\mathrm{Hz}$ with an increase in frequency with age (Marshall et al., 2002; Stroganova et al., 1999). Therefore, the timefrequency range for the analyses was chosen for each age group separately based on visual inspection of the differences between conditions. Mean amplitude of P3, Pz, P4, O1, and 02 served as the dependent variable. Consistent with the literature, the selected frequencies

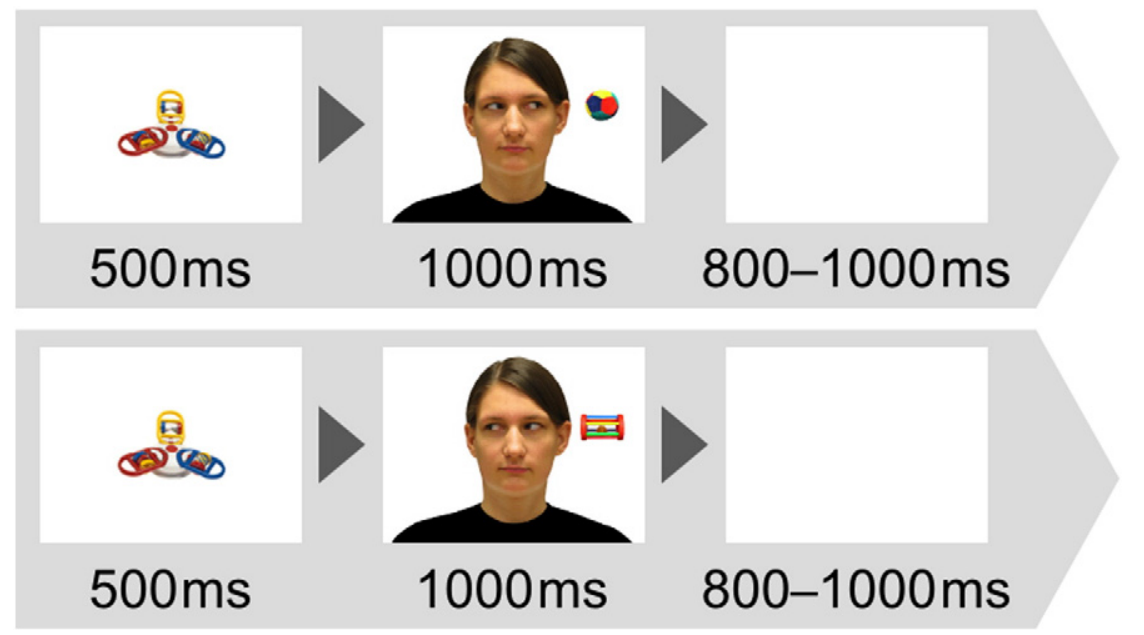

Fig. 1. Examples of a trial in the object-directed condition (top) and the object-averted condition (bottom). 
increased with age (Marshall et al., 2002; Stroganova et al., 1999). See Table 3 in the results section for an overview of the time-frequency ranges.

As no differences between channels are expected, the amplitude of the frontal channels F3, Fz, F4, FC3, and FC4 was averaged for theta activity and the amplitude of the posterior-occipital channels P3, Pz, P4, O1, and $\mathrm{O} 2$ was averaged for alpha activity for each condition. The two conditions were contrasted using paired $t$-tests separately for each age group. $P$ values are Bonferroni-Holm corrected.

\section{Results}

Theta

No significant differences between conditions were found for the 2-, $4-$, and 9-month-olds, all ps $>.431$. However, the object-averted condition and the object-directed condition differed significantly in the 5month-olds, $t(15)=-3.50, p=.012$. Theta synchronized more in the object-averted compared to the object-directed condition. Theta

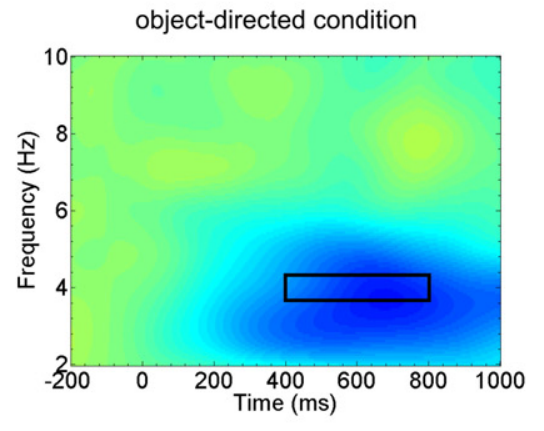

object-directed condition
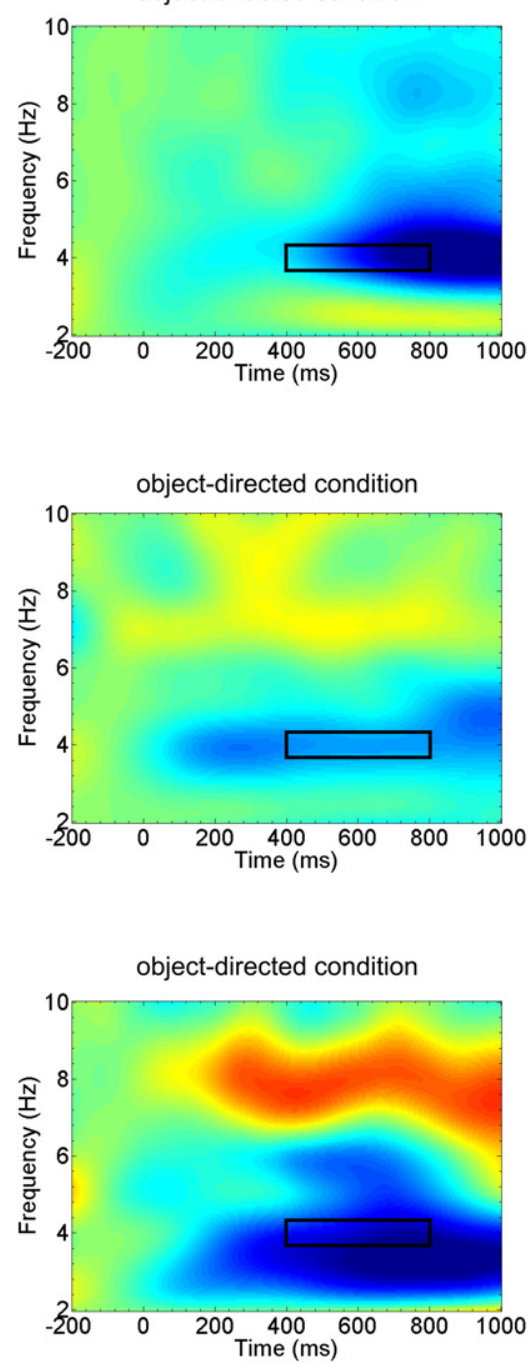

\section{2 months}

object-averted condition

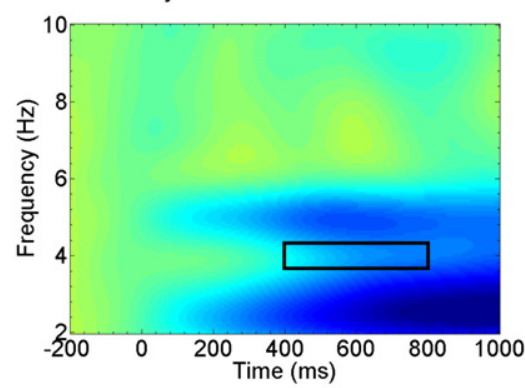

4 months

object-averted condition

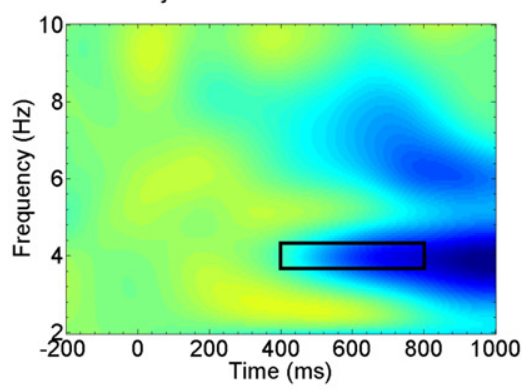

5 months

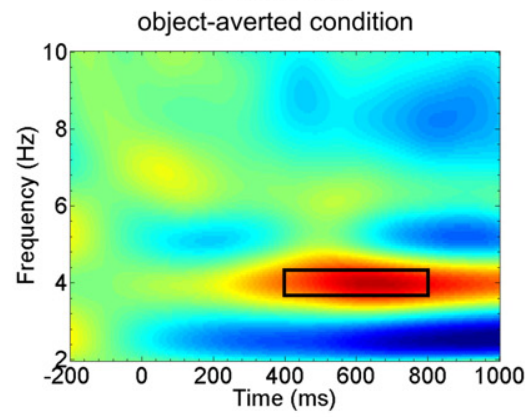

9 months

object-averted condition

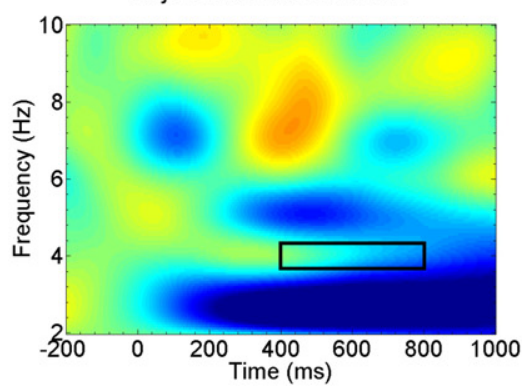

object-directed condition - object-averted condition

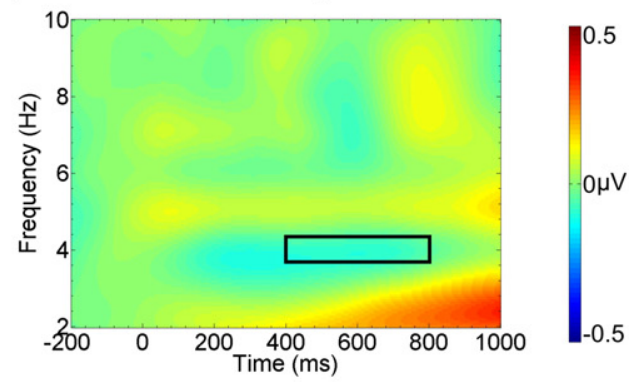

object-directed condition - object-averted condition

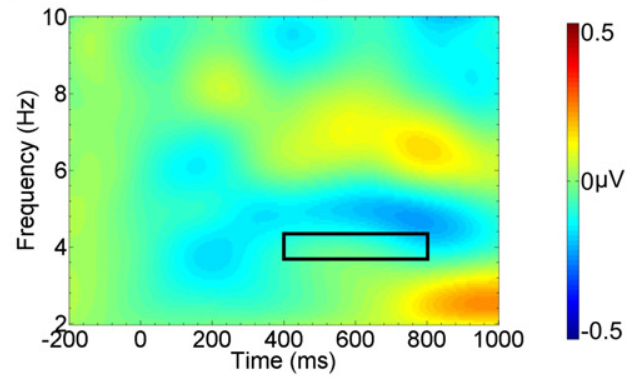

object-directed condition - object-averted condition

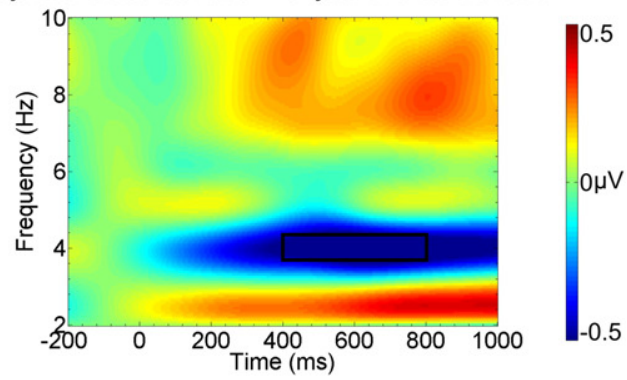

object-directed condition - object-averted condition

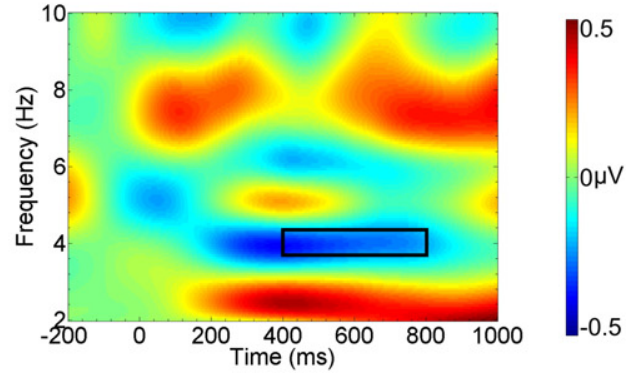

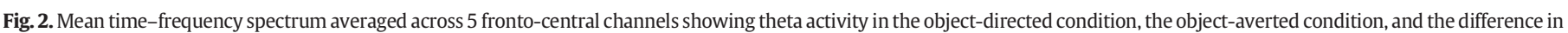
theta activity between the object-directed-object-averted condition in 2-, 4-, 5-, and 9-month-olds. The rectangle marks the analyzed time window at $4 \mathrm{~Hz}$. 
activity in both conditions did not differ from baseline, all $p s>.195$. See Fig. 2 and Table 2 for means and standard errors.

Alpha

Whereas alpha activity in the object-directed and the object-averted condition was not different in the 2- and 5-month age groups (all ps > .619), there were significant differences between the conditions at the ages of 4 and 9 months $(t(15)=-3.46, p=.008$ for the 4-montholds, $t(11)=-2.73, p=.038$ for the 9-month-olds). While both conditions in both age groups differed significantly from baseline (4-month-olds: $t(15)=-7.22, p<.001$ for the object-directed condition and $t(15)=-3.65, p=.006$ for the object-averted condition; 9-month-olds: $t(11)=-6.01, p<.001$ for the object-directed condition and $t(11)=-3.50, p=.015$ for the object-averted condition), the desynchronization was enhanced in the object-directed compared to the object-averted condition at both ages. See Fig. 3 and Table 3 for an overview of the means and standard errors.

\section{Discussion}

In order to investigate developmental changes in neural mechanisms underlying the processing of eye gaze-object relations in early infancy, we presented infants (2, 4, 5, and 9 months old) with faces that were either looking away from or toward objects while EEG was measured. Differences between conditions in the theta and the alpha frequency bands were investigated for each age group. In line with studies showing that $4-8$-month-old infants differentiate between object-directed and object-averted gaze shifts with regard to looking times and ERPs (Hoehl et al., 2008, 2009; Senju et al., 2008), we have shown that theta and alpha oscillations are sensitive measures to investigate this social cognitive ability in these age groups.

Theta synchronization is suggested to reflect the involvement of an executive attention network and internal attentional processes (Bazhenova et al., 2007; Orekhova et al., 1999). We expected theta activity to alter with the development of this network. We found differences between conditions only in the 5-month-old infants. At that age, this attention network is thought to develop (Mundy and Newell, 2007). Theta activity synchronized more in the object-averted than in the object-directed condition. It is important to note that theta activity in the 5-month-olds did not differ from baseline. The difference in theta synchronization between conditions must therefore be interpreted very cautiously.

Alpha desynchronization has been shown to be sensitive to attentional mechanisms that enable the brain to suppress irrelevant input and focus on relevant information in adults (Ward, 2003). Cues such as eye gaze signal objects that can be relevant for a beholder (Frischen et al., 2007; George and Conty, 2008; Hoehl et al., 2009; Senju and Johnson, 2009). Enhanced alpha desynchronization may reflect the attentive processing of such information. We speculate that it, as such, enables or facilitates early social learning mechanisms in infants. This is in line with studies that relate alpha desynchronization to joint attention in infants and adults (Hoehl et al., 2014a; Lachat et al., 2012). As infants at 4 months of age are already sensitive to looker-object relations and use eye gaze for facilitated object learning (e.g., Reid et al., 2004), we expected alpha desynchronization in response to object-directed eye gaze from 4 months onwards. This expectation was partly fulfilled as alpha desynchronized more in the object-directed condition in 4and 9-month-olds, but not at 2 and 5 months of age. Taken together with the results on theta activity, substantial developmental changes in the neural processing of object-looker relation have been detected in the current study.

As we did not find a difference between conditions on both frequency bands at 2 months of age, we can only speculate about the neural processes occurring at this age. Infants at this age show nearly no overt gaze following behavior. The tendency to follow another person's gaze, and, therefore, the ability to detect object-looker relations, develops between 2 and 4 months of age (Gredebäck et al., 2010). Thus, infants at 2 months of age might simply not detect differences between conditions. Alternatively, it is possible that infants are able to differentiate between the conditions but our methodology was not capable of detecting this. In line with the current results, no ERP effects have been observed using the same stimuli in 2-month-olds (Hoehl et al., 2009).

At 4 months of age, infants showed enhanced alpha desynchronization in the object-directed compared to the object-averted condition. Alpha desynchronization is a sensitive measure for attentional mechanisms that suppress irrelevant information and therefore focus attention on relevant information (Ward, 2003). Social cues such as eye gaze or head turn can guide infants' attention and can lead to enhanced memory encoding of cued objects in 4-month-olds (Hoehl et al., 2014b; Hood et al., 1998; Reid and Striano, 2005; Reid et al., 2004; Wahl et al., 2013). Thus, alpha desynchronization in the object-directed condition may reflect focused attention to gaze cued objects and thereby be related to social learning processes (Hoehl et al., 2014a).

At the same age, no difference between conditions was found in the theta range. So far, studies that have related theta synchronization to attentional processes have all investigated slightly older infants (Bazhenova et al., 2007; Orekhova et al., 1999, 2006; Stroganova et al., 1998). Theta synchronization has nonetheless been discussed to be related to the involvement of an attention network that is responsible for the executive control of attention that emerges between 4 and 6 months of age (Bazhenova et al., 2007; Mundy and Newell, 2007; Orekhova et al., 1999). As attention is thought to be guided automatically by social cues at four months, it is possible that this attention network is not yet involved in processing social cues in our sample.

Similar to 4-month-old infants, 9-month-olds also showed enhanced alpha desynchronization in the object-directed condition but their theta activity did not differ between conditions. At that age, infants are able to use joint attention interactions for enhanced object processing (Cleveland et al., 2007; Striano et al., 2006) and alpha desynchronization has been observed in joint attention interactions (Hoehl et al., 2014a). As in the 4-month-olds, eye gaze direction in the object-directed condition may guide infants' attention to a relevant object, thus attention is focused on that object and alpha desynchronization could reflect these processes. In comparison to the younger age group, 9-month-olds are increasingly able to monitor their own and another person's attention (Mundy and Newell, 2007). This additional skill improves the infant's ability to detect and analyze the looker-object relationship and thereby to differentiate between object-directed and object-averted eye gaze. However, even in this older age group, it is likely that automatic shifts of attention are still part of gaze cueing effects as it is known that they still exist in typically developing children and in adults (Friesen et al., 2004; Senju et al., 2004). Alpha desynchronization during infancy

Table 2

Overview of the time range, the frequency and descriptive statistics of the analyses of theta activity.

\begin{tabular}{|c|c|c|c|c|}
\hline Theta & 2-month-olds & 4-month-olds & 5-month-olds & 9-month-olds \\
\hline Time range & $400-800 \mathrm{~ms}$ & $400-800 \mathrm{~ms}$ & $400-800 \mathrm{~ms}$ & $400-800 \mathrm{~ms}$ \\
\hline Frequency & $4 \mathrm{~Hz}$ & $4 \mathrm{~Hz}$ & $4 \mathrm{~Hz}$ & $4 \mathrm{~Hz}$ \\
\hline Mean (standard error) object-directed condition $[\mu \mathrm{V}]$ & $-0.32(0.08)$ & $-0.37(0.08)$ & $-0.23(0.15)$ & $-0.44(0.18)$ \\
\hline Mean (standard error) object-averted condition $[\mu \mathrm{V}]$ & $-0.24(0.11)$ & $-0.30(0.11)$ & $0.38(0.19)$ & $-0.14(0.29)$ \\
\hline
\end{tabular}


could potentially relate to social object learning guided by the mechanisms that are present at each specific age: automatic cueing of attention in 4-month-olds and, additionally, more volitionally controlled shifts of attention at 9 months of age. It is worth highlighting that in 4- and 9month-olds, alpha desynchronized when compared to baseline in both conditions. This might be due to both conditions conveying information about an object-looker relation, but it is only in the object-directed condition that eye gaze direction and object location match. This matching enables the infant to relate another person's eye gaze to the object, which may lead to a focusing of attention on this stimulus. This, in turn, might trigger processes similar to those found in adults in situations with mutual attentiveness (Lachat et al., 2012) that are reflected in enhanced alpha desynchronization. No difference in theta activity was found in the 9-month-olds. As joint attention skills are better developed at that age and the attention network matures, we assume that infants can easily detect differences between conditions without or with less effort of an internal control of attention (Orekhova et al., 1999).

Whereas 4- and 9-month-olds show no difference in theta activity but exhibit an enhanced alpha desynchronization, 5-month-olds show the reversed pattern: theta activity differed between conditions with

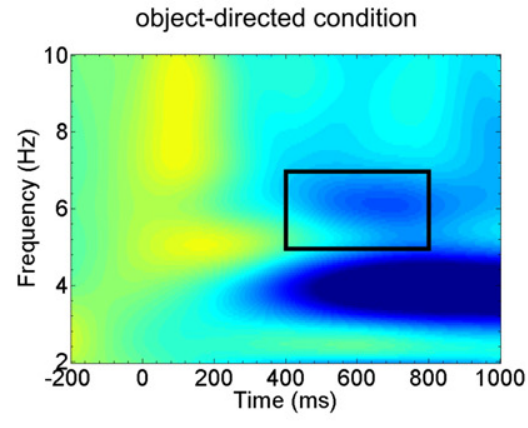

object-directed condition
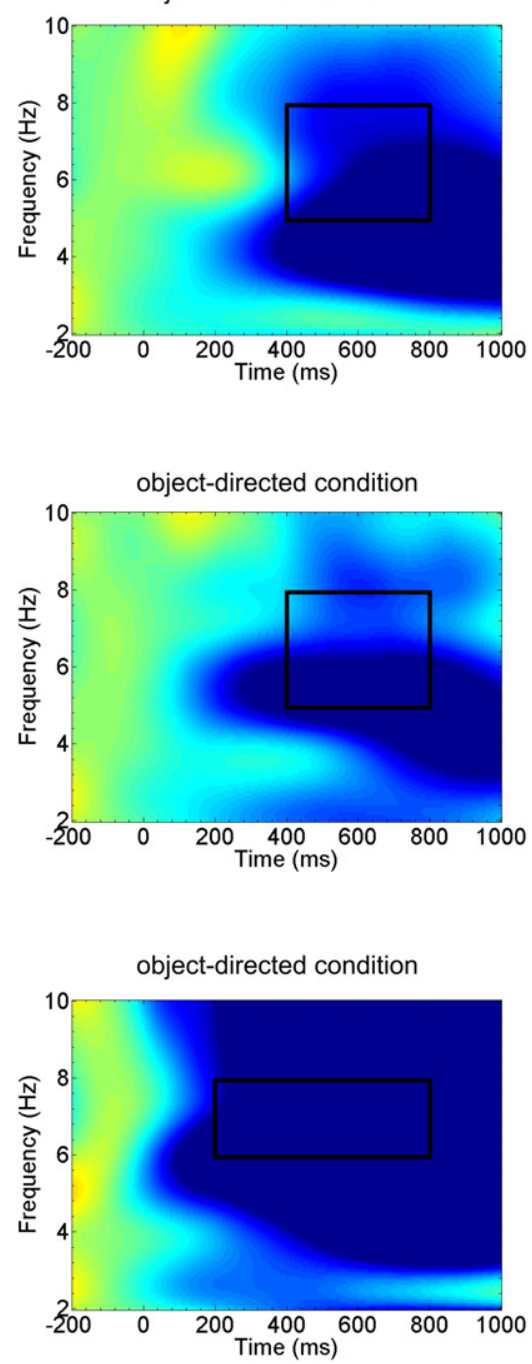

\section{2 months}

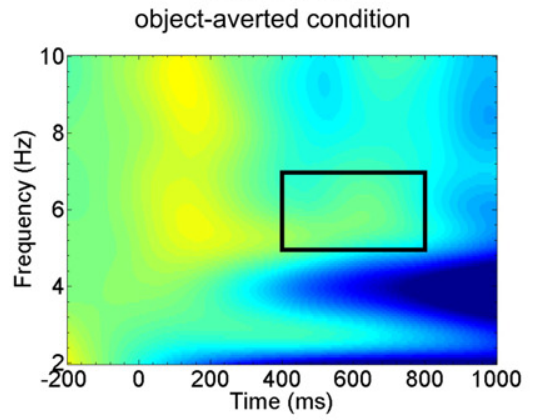

4 months

object-averted condition

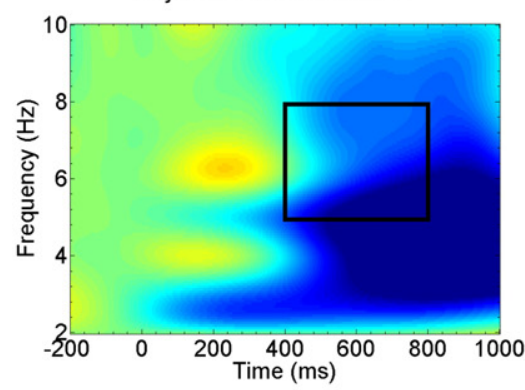

\section{5 months}

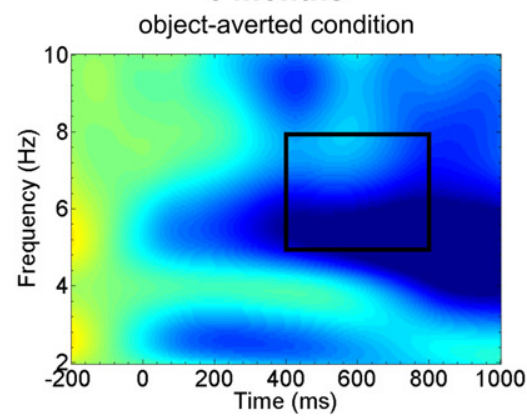

9 months

object-averted condition

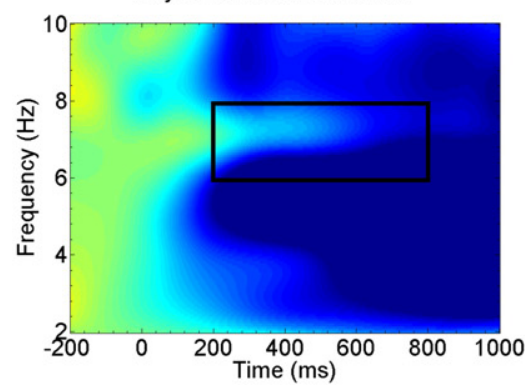

object-directed condition - object-averted condition

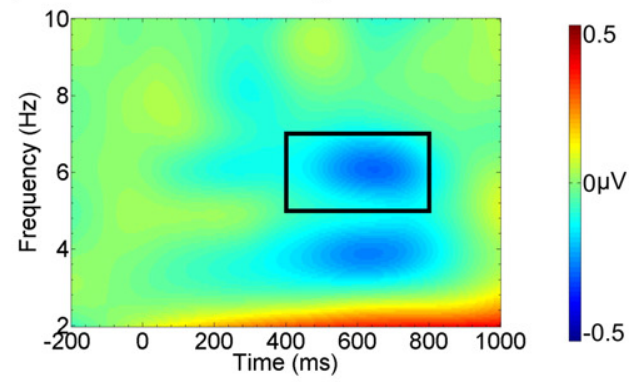

object-directed condition - object-averted condition

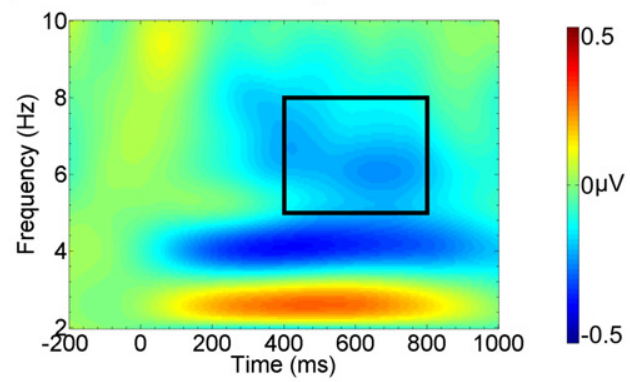

object-directed condition - object-averted condition

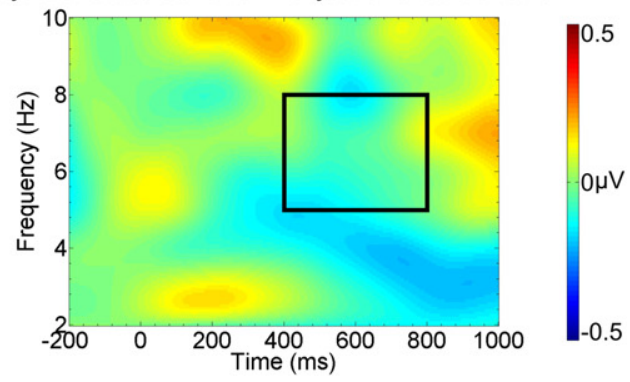

object-directed condition - object-averted condition

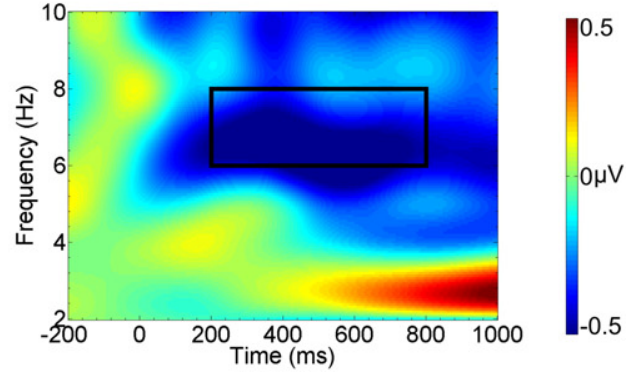

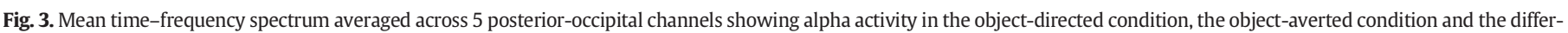
ence in alpha activity between the object-directed-object-averted condition in 2-, 4-, 5-, and 9-month-olds. The rectangle marks the analyzed time-frequency range. 
Table 3

Overview of the time and frequency ranges and descriptive statistics of the analyses of alpha activity.

\begin{tabular}{|c|c|c|c|c|}
\hline Alpha & 2-month-olds & 4-month-olds & 5-month-olds & 9-month-olds \\
\hline Time range & $400-800 \mathrm{~ms}$ & $400-800 \mathrm{~ms}$ & $400-800 \mathrm{~ms}$ & $200-800 \mathrm{~ms}$ \\
\hline Frequency range & $5-7 \mathrm{~Hz}$ & $5-8 \mathrm{~Hz}$ & $5-8 \mathrm{~Hz}$ & $6-8 \mathrm{~Hz}$ \\
\hline Mean (standard error) object-directed condition $[\mu \mathrm{V}]$ & $-0.22(0.08)$ & $-0.53(0.07)$ & $-0.46(0.17)$ & $-0.89(0.15)$ \\
\hline Mean (standard error) object-averted condition $[\mu \mathrm{V}]$ & $-0.07(0.09)$ & $-0.34(0.09)$ & $-0.40(0.15)$ & $-0.47(0.13)$ \\
\hline
\end{tabular}

enhanced theta synchronization in response to object-averted eye gaze, but alpha-band activity did not.

Why do the 5-month-olds differ in their response from the 4- and 9month-olds? The attention network, being related to theta synchronization, is assumed to develop precisely at that age (Mundy and Newell, 2007). Furthermore, at the same age, gaze following abilities and joint attention skills improve but are not yet fully developed (Gredebäck et al., 2010; Striano and Bertin, 2005). Moreover, the ability to use a joint attention context to learn about objects develops (Cleveland et al., 2007) and the reaction to social cues is changing from automatic shifts of attention to additional voluntary mechanisms. Five-monthold infants are just developing social abilities and might, therefore, be extremely sensitive to social cues and also to the disrupted relation between object and eye gaze in the object-averted condition. Thus, this condition may require more attentional control. In line with ERP results showing that only attentional processes and not memory processes are affected when a disturbed looker-object relation is presented to infants at that age (Hoehl et al., 2009), differences in theta activity but not in alpha were found in the current study.

Here, we investigated how processing of object-directed and objectaverted eye gaze develops during infancy measuring oscillatory brain activity. While alpha desynchronization in 4- and 9-month-olds is probably reflecting focused attention that may enable early social learning processes, theta synchronization at 5 months may reflect the development of an executive attention network, and therefore, the transition from a rather automatic shift of attention in reaction to social cues to an enhanced deliberate control. The interplay between alpha- and theta-band activities represents striking developmental changes in infants' neural processing of social information. Future research is needed to investigate whether the differences in oscillatory brain activity are indeed related to the encoding or learning of new information.

\section{Acknowledgments}

We are grateful to the children and parents who participated. This work was supported by a grant from the Deutsche Forschungsgemeinschaft (DFG) [grant number HO 4342/2-2] awarded to Stefanie Hoehl and a stipend from the Studienstiftung des deutschen Volkes awarded to Christine Michel. Eugenio Parise and Vincent Reid are researchers in the International Centre for Language and Communicative Development (LuCiD) at Lancaster University. The support of the Economic and Social Research Council [ES/L008955/1] is gratefully acknowledged.

\section{References}

Bazhenova, O.V., Stroganova, T.A., Doussard-Roosevelt, J.A., Posikera, I.A., Porges, S.W., 2007. Physiological responses of 5-month-old infants to smiling and blank faces. Int. J. Psychophysiol. 63 (1), 64-76.

Bertenthal, B.I., Boyer, T.W., Harding, S., 2014. When do infants begin to follow a point? Dev. Psychol. 50 (8), 2036-2048. http://dx.doi.org/10.1037/a0037152.

Cleveland, A., Striano, T., 2007. The effects of joint attention on object processing in 4- and 9-month-old infants. Infant Behav. Dev. 30 (3), 499-504. http://dx.doi.org/10.1016/j. infbeh.2006.10.009.

Cleveland, A., Schug, M., Striano, T., 2007. Joint attention and object learning in 5- and 7month-old infants. Infant Child Dev. 16 (3), 295-306. http://dx.doi.org/10.1002/icd.508.

Csibra, G., Gergely, G., 2006. Social learning and social cognition: The case for pedagogy. In: Munakata, Y., Johnson, M.H. (Eds.), Processes of Change in Brain and Cognitive Development: Attention and Performance XXI. University Press, Oxford, pp. 249-274.
Csibra, G., Davis, G., Spratling, M.W., Johnson, M.H., 2000. Gamma oscillations and object processing in the infant brain. Science 290 (5496), 1582-1585. http://dx.doi.org/10. 1126/science.290.5496.1582.

Elsabbagh, M., Volein, A., Csibra, G., Holmboe, K., Garwood, H., Tucker, L., Johnson, M.H 2009. Neural correlates of eye gaze processing in the infant broader autism phenotype. Biol. Psychiatry 65 (1), 31-38. http://dx.doi.org/10.1016/j.biopsych.2008.09.034.

Friesen, C.K., Ristic, J., Kingstone, A., 2004. Attentional effects of counterpredictive gaze and arrow cues. J. Exp. Psychol. Hum. Percept. Perform. 30 (2), 319-329. http://dx doi.org/10.1037/0096-1523.30.2.319.

Frischen, A., Bayliss, A.P., Tipper, S.P., 2007. Gaze cueing of attention: Visual attention, social cognition, and individual differences. Psychol. Bull. 133 (4), 694-724. http://dx. doi.org/10.1037/0033-2909.133.4.694.

George, N., Conty, L., 2008. Facing the gaze of others. Neurophysiol. Clin. 38 (3), 197-207. http://dx.doi.org/10.1016/j.neucli.2008.03.001.

Gredebäck, G., Fikke, L., Melinder, A., 2010. The development of joint visual attention: A longitudinal study of gaze following during interactions with mothers and strangers. Dev. Sci. 13 (6), 839-848. http://dx.doi.org/10.1111/j.1467-7687.2009.00945.x.

Hoehl, S., Reid, V., Mooney, J., Striano, T., 2008. What are you looking at? Infants' neural processing of an adult's object-directed eye gaze. Dev. Sci. 11 (1), 10-16.

Hoehl, S., Reid, V.M., Parise, E., Handl, A., Palumbo, L., Striano, T., 2009. Looking at eye gaze processing and its neural correlates in infancy-Implications for social development and autism spectrum disorder. Child Dev. 80 (4), 968-985. http://dx.doi.org/10. 1111/j.1467-8624.2009.01311.x.

Hoehl, S., Michel, C., Reid, V.M., Parise, E., Striano, T., 2014a. Eye contact during live social interaction modulates infants' oscillatory brain activity. Soc. Neurosci. 9 (3), 300-308. http://dx.doi.org/10.1080/17470919.2014.884982.

Hoehl, S., Wahl, S., Pauen, S., 2014b. Disentangling the effects of an adult model's eye gaze and head orientation on young infants' processing of a previously attended object. Infancy 19 (1), 53-64. http://dx.doi.org/10.1111/infa.12035.

Hood, B.M., Willen, J.D., Driver, J., 1998. Adult's eyes trigger shifts of visual attention in human infants. Psychological Science 9(2), pp. 131-134. http://dx.doi.org/10.1111/ 1467-9280.00024.

Klimesch, W., 1999. EEG alpha and theta oscillations reflect cognitive and memory performance: a review and analysis. Brain Res. Rev. 29 (2-3), 169-195. http://dx.doi.org/10. 1016/S0165-0173(98)00056-3.

Lachat, F., Hugueville, L., Lemaréchal, J.-D., Conty, L., George, N., 2012. Oscillatory brain correlates of live joint attention: A dual-EEG study. Front. Hum. Neurosci. 6. http:// dx.doi.org/10.3389/fnhum.2012.00156.

Marshall, P.., Bar-Haim, Y., Fox, N.A., 2002. Development of the EEG from 5 months to 4 years of age. Clin. Neurophysiol. 113 (8), 1199-1208. http://dx.doi.org/10.1016/ S1388-2457(02)00163-3.

Moore, C., Corkum, V., 1998. Infant gaze following based on eye direction. Br. J. Dev. Psychol. 16 (4), 495-503. http://dx.doi.org/10.1111/j.2044-835X.1998.tb00767.x.

Mundy, P., Newell, L., 2007. Attention, joint attention, and social cognition. Curr. Dir. Psychol. Sci. 16 (5), 269-274. http://dx.doi.org/10.1111/j.1467-8721.2007.00518.x.

Nelson, C.A., 1997. Electrophysiological correlates of memory development in the first year of life. In: Reese, H.W., Franzen, M.D. (Eds.), Biological and Neuropsychological Mechanisms: Life-Span Developmental Psychology. Lawrence Erlbaum Associates, Inc., Hillsdale, NJ England, pp. 95-131.

Orekhova, E.V., Stroganova, T.A., Posikera, I.N., 1999. Theta synchronization during sustained anticipatory attention in infants over the second half of the first year of life. Int. J. Psychophysiol. 32 (2), 151-172.

Orekhova, E.V., Stroganova, T.A., Posikera, I.N., Elam, M., 2006. EEG theta rhythm in infants and preschool children. Clin. Neurophysiol. 117 (5), 1047-1062.

Parise, E., Csibra, G., 2013. Neural responses to multimodal ostensive signals in 5-monthold infants. PLoS One 8 (8), e72360. http://dx.doi.org/10.1371/journal.pone.0072360.

Petersen, S.E., Posner, M.I., 2012. The attention system of the human brain: 20 years after Annu. Rev. Neurosci. 35, 73-89.

Posner, M.I., Petersen, S.E., 1990. The attention system of the human brain. Annu. Rev. Neurosci. 13, 25-42.

Reid, V.M., Striano, T., 2005. Adult gaze influences infant attention and object processing: Implications for cognitive neuroscience. Eur. J. Neurosci. 21 (6), 1763-1766. http:// dx.doi.org/10.1111/j.1460-9568.2005.03986.x.

Reid, V.M., Striano, T., 2007. The directed attention model of infant social cognition. Eur. J. Dev. Psychol. 4 (1), 100-110.

Reid, V.M., Striano, T., Kaufman, J., Johnson, M.H., 2004. Eye gaze cueing facilitates neural processing of objects in 4-month-old infants. NeuroReport 15 (16), 2553-2555. http://dx.doi.org/10.1097/00001756-200411150-00025.

Reynolds, G.D., Richards, J.E., 2005. Familiarization, attention, and recognition memory in infancy: An event-related potential and cortical source localization study. Dev. Psychol. 41 (4), 598-615. http://dx.doi.org/10.1037/0012-1649.41.4.598.

Saby, J.N., Marshall, P.J., 2012. The utility of EEG band power analysis in the study of infancy and early childhood. Dev. Neuropsychol. 37 (3), 253-273. http://dx.doi.org/10 1080/87565641.2011.614663. 
Senju, A., Johnson, M.H., 2009. The eye contact effect: Mechanisms and development. Trends Cogn. Sci. 13 (3), 127-134. http://dx.doi.org/10.1016/j.tics.2008.11.009.

Senju, A., Tojo, Y., Dairoku, H., Hasegawa, T., 2004. Reflexive orienting in response to eye gaze and an arrow in children with and without autism. J. Child Psychol. Psychiatry 45 (3), 445-458. http://dx.doi.org/10.1111/j.1469-7610.2004.00236.x.

Senju, A., Csibra, G., Johnson, M.H., 2008. Understanding the referential nature of looking: Infants' preference for object-directed gaze. Cognition 108 (2), 303-319. http://dx. doi.org/10.1016/j.cognition.2008.02.009.

Southgate, V., Csibra, G., Kaufman, J., Johnson, M.H., 2008. Distinct processing of objects and faces in the infant brain. J. Cogn. Neurosci. 20 (4), 741-749. http://dx.doi.org/ 10.1162/jocn.2008.20052.

Striano, T., Bertin, E., 2005. Social-cognitive skills between 5 and 10 months of age. Br. J. Dev. Psychol. 23 (4), 559-568.

Striano, T., Stahl, D., 2005. Sensitivity to triadic attention in early infancy. Dev. Sci. 8 (4), 333-343. http://dx.doi.org/10.1111/j.1467-7687.2005.00421.x

Striano, T., Reid, V.M., Hoehl, S., 2006. Neural mechanisms of joint attention in infancy. Eur. J. Neurosci. 23 (10), 2819-2823. http://dx.doi.org/10.1111/j.1460-9568.2006. 04822.x.
Stroganova, T.A., Orekhova, E.V., 2007. EEG and infant states. In: de Haan, M. (Ed.), Infant EEG and Event-Related Potentials. Psychology Press, New York, NY, US, pp. 251-287. Stroganova, T.A., Orekhova, E.V., Posikera, I.N., 1998. Externally and internally controlled attention in infants: An EEG study. Int. J. Psychophysiol. 30 (3), 339-351.

Stroganova, T.A., Orekhova, E.V., Posikera, I.N., 1999. EEG alpha rhythm in infants. Clin. Neurophysiol. 110 (6), 997-1012.

Wahl, S., Michel, C., Pauen, S., Hoehl, S., 2013. Head and eye movements affect object processing in 4-month-old infants more than an artificial orientation cue. Br. J. Dev. Psychol. 31 (2), 212-230. http://dx.doi.org/10.1111/bjdp.12001.

Ward, L.M., 2003. Synchronous neural oscillations and cognitive processes. Trends Cogn. Sci. 7 (12), 553-559. http://dx.doi.org/10.1016/j.tics.2003.10.012.

Webb, S.J., Long, J.D., Nelson, C.A., 2005. A longitudinal investigation of visual eventrelated potentials in the first year of life. Dev. Sci. 8 (6), 605-616. http://dx.doi.org/ 10.1111/j.1467-7687.2005.00452.x. 